\section{SCAN YOUR IMPLANT BAR CODES}

iChiropro from Bien-Air is now supplied with the Apple Lightning connection as standard as well as the iPad Air Adaptor and iPad 4 adaptor to make your working life even easier.

iPad 4 and iPad Air have the higher resolution camera, which enables users to scan the implant bar code of most implants. The intuitive bar code scanner is just one of the many innovative features of the iChiropro App.

The iChiropro by Bien-Air is a revolutionary physiodispenser, controlled from an iPad. Simple to use, the intuitive, ergonomic application ensures significant time savings during opera-

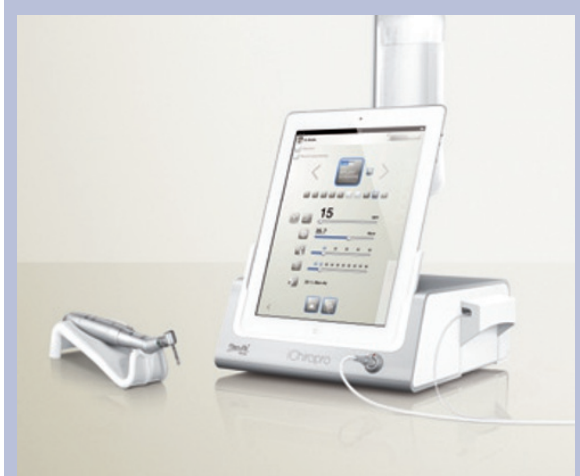

tions. Available free from the Apple App Store, the iChiropro application opens up numerous avenues in terms of customisation options. New innovative functions are added approximately every 6-10 months, to guarantee users a simple, ergonomic and up-to-date experience.

The most recent App update includes pre-planning of patients' implant placements, the ability to record the placement of multiple implants in the patient file and a new look and feel to the application.

The multi-user iChiropro incorporates the complete sequences of the leading implant manufacturers with their predefined settings. It also offers the option of saving and exporting operation data, in the form of graphics and tables, attached to the patient file built into the control interface. It's the only device which allows users to save, export and print all operation parameters, whilst documenting these with the patient data and the implants used. The application allows multiple users to customise and store their own sequences.

For more details or an in-surgery demonstration, telephone Bien-Air on 01293 550200 or email ba-uk@bienair.com.

\section{SAY GOODBYE TO GAPPY GRINS}

The aim of the 'Goodbye Gaps' programme is to get one million people with missing teeth into dental practices to discuss implant treatment. This initiative expands and amplifies the extensive support already offered by DENTSPLY Implants to help dental professionals gain more fee income from treating implant cases.

The Goodbye Gaps campaign improves patient access to dentists who place implants, through direct contact as well as via referral by their own general practitioner. The information available to patients about implant treatment is also being increased, through national and local, online and print media.

General dental practitioners who want to begin restoring implant cases for their own patients, can get started by attending 'R£LAX' events hosted by local surgical partners across the country. The 'R£LAX' package includes free training and a mini prosthetics kit with which to restore the first implant case. DENTSPLY Implants

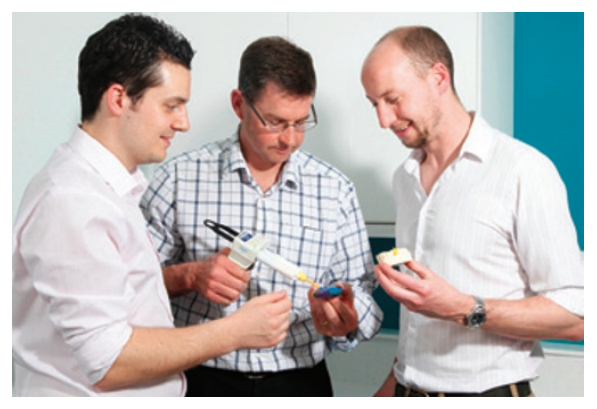

also offers dentists who restore implant cases chairside literature and merchandising to help acquaint their patients.

Further training is available to clinicians wishing to learn to restore more complex cases. This is backed by local clinical support from surgical partners. In-practice marketing advice and support is also available through DENTSPLY Implants' nationwide network of local territory managers.

Dental professionals who want to benefit from the 'Goodbye Gaps' campaign can find further details of the support provided by DENTSPLY Implants by visiting www.dentists4implants.com/goodbyegaps. 